\title{
Padre preparado y comprometido en su rol de acompañante durante el proceso de parto
}

\begin{abstract}
Temática: promoción y prevención.
Aporte a la disciplina: al conocer los esfuerzos por mejorar la calidad asistencial en el proceso de parto, cuando se trata de implementar acciones de promoción de la salud que se perpetúen en el tiempo, ocurre que un proceso relativamente simple y fisiológico muchas veces se transforma en algo complejo en su abordaje. El estudio a través de la investigación-acción permite desarrollar un trabajo de sistema, ya que mediante intervenciones complejas e integradoras, se promueve la comprensión del beneficio de la participación, la motivación y el compromiso para llevarla a cabo. Desde la mirada sistémica, es imprescindible la participación comprometida de los distintos actores (el padre, la madre y el equipo de salud) porque permite la promoción de acciones con miras al desarrollo de una paternidad saludable y, en consecuencia, de familias comprometidas con sus hijos y con su crianza. Algunas iniciativas actuales de preparación de los padres para el parto y para el nacimiento se han focalizado en las necesidades de la madre y de su hijo para tener una maternidad segura y con bienestar. Para esta última década, el desafío es involucrar al padre en el nacimiento de manera efectiva.
\end{abstract}

\section{RESUMEN}

Objetivo: identificar las necesidades de preparación del padre acompañante para brindar apoyo a su pareja durante el proceso de parto. Método: metodología cualitativa: investigación-acción con la participación de doce padres y sus parejas, con quienes se exploraron las necesidades educativas por medio de grupos focales y entrevistas estructuradas. Se desarrolló, implementó y evaluó la preparación antenatal del padre en su rol de acompañante durante los años 2015 y 2016. Resultados: una expectativa que se constituyó en un gran tema emergente fue "conocer más acerca del proceso de parto" y "estar ahí con ella y para ella", como beneficio para su rol de acompañante preparado y sensible a las necesidades de la mujer. Conclusión: es indispensable considerar estrategias que fortalezcan el rol del padre y aumenten sus competencias para la integración, empoderamiento y acompañamiento durante el proceso de parto.

PALABRAS CLAVE (FUENTE: DeCS)

Educación prenatal; nacimiento; parto; padre; apoyo social; investigación-acción; investigación en servicios de salud.

\section{DOI: 10.5294/aqui.2018.18.4.4}

Para citar este artículo / To reference this article / Para citar este artigo Muñoz-Serrano M, Uribe-Torres C, Hoga L. Padre preparado y comprometido en su rol de acompañante durante el proceso de parto. Aquichan. 2018; 18(4): 415-425. DOI: 10.5294/aqui.2018.18.4.4

\footnotetext{
1 orcid.org/0000-0001-9104-3687. Escuela de Enfermería, Pontificia Universidad Católica de Chile, Chile. mmunozse@uc.cl

$2 \bowtie$ orcid.org/0000-0002-0699-7695. Escuela de Enfermería, Pontificia Universidad Católica de Chile, Chile. curibet@uc.cl

3 orcid.org/0000-0002-6433-5307. Escola de enfermagem, Universidade de São Paulo, Brasil. kikatuca@usp.br
}

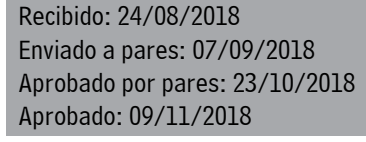




\section{Fathers Prepared For and Committed to Their Role as Companions during the Birth Process}

\section{ABSTRACT}

Objective: To identify fathers' needs in their preparation as a companion to provide emotional support to mothers during childbirth. Materials and methods: An action research project was carried out involving twelve men and their wives. Their educational needs were explored using focus groups and structured interviews during 2015 and 2016. Results: The main emerging themes were "knowing more about the birth process" and "being there with her and for her" as benefits that contribute to the role of a sensitive companion prepared to understand the woman's needs. Considering one of the major emerging themes, antenatal training for fathers as companions and support during childbirth was developed, implemented and evaluated. Conclusion: It is essential to consider different strategies to strengthen the father's role by increasing their skills for integration, empowerment and support during birth, and to enhance their satisfaction in meeting the needs felt in a family birth scenario.

\section{KEYWORDS (SOURCE: DeCS)}

Prenatal education; childbirth; parturition; fathers; social support; action research; health services research. 


\section{Pai preparado e comprometido em seu papel como acompanhante durante o processo de nascimento}

\section{RESUMO}

Objetivo: identificar as necessidades de preparação do pai acompanhante para oferecer suporte e continência para a sua parceira durante o processo de parto. Método: trata-se de uma metodologia qualitativa de pesquisa, que contou com a participação de 12 pais e suas parceiras, com os quais foram exploradas as necessidades educativas, por meio da utilização de técnicas de grupo focal e entrevistas estruturadas. Foi desenvolvida, implementada e avaliada a preparação pré-natal para o desempenho de seus papéis de acompanhante durante 2015 e 2016. Resultados: expectativas que se constituíram como grandes temas emergentes foram "conhecer mais sobre o processo de parto" e "estar aî́ com ela e para ela" como benefícios para seus papéis de acompanhantes preparados e sensíveis às necessidades da mulher. Conclusão: é indispensável considerar estratégias que permitam relevar o papel do pai, aumentando suas competências para a integração, empoderamento e acompanhamento durante o processo de parto, para promover a satisfação da necessidade sentida de um genuíno nascimento em família.

PALAVRAS-CHAVE (FONTE: DECS)

Educação pré-natal; nascimento; parto; pai; apoio social; pesquisa ação; pesquisa sobre serviços de saúde. 


\section{Introducción}

Con el objetivo de alcanzar el cuidado integral del nacimiento, el desafío hoy, en el mundo, es considerar e involucrar al padre en el nacimiento de su hijo, para que este se constituya en un participante activo, tanto en el apoyo a la mujer y contención materna durante el nacimiento (1), como en acciones de promoción de vinculación con su hijo recién nacido (2). El modelo actual de atención, principalmente en Latinoamérica, considera las prácticas de participación paterna de una manera hegemónica y directiva, con escasa o nula preparación previa, lo que puede generar situaciones de estrés y ambivalencia emocional en los varones (3).

La preparación requerida para promover los cambios en la actitud de los padres está distante de lo que puede aportar la intervención de la salud tradicional, en la que la educación se transfiere de forma vertical y los participantes asumen un rol pasivo (4). Asimismo, algunos autores han hecho explícita la recomendación de desarrollar propuestas de intervenciones especiales para los padres (5-7). Esta preparación dirigida al acompañamiento y soporte de la mujer para el parto aparece en los estudios reportados como una necesidad de preparación elemental y prioritaria $(5,8-10)$. No obstante, el padre continua siendo visibilizado en un lugar secundario y pasivo, como espectador del parto de la mujer (10), y, por ende, cuando este manifiesta sus demandas, las plantea para cumplir ese rol a partir de las necesidades maternas (6, $10,11)$. Un verdadero rol de acompañante, para "ayudar a parir" o "parir junto a su mujer" $(7,12)$, debe enmarcarse desde la satisfacción que se genera en el padre acompañante cuando siente que ha sido útil al brindar apoyo físico para el alivio del dolor, así como fuerzas a la mujer, todo desde una experiencia particular e individual como padre-acompañante y con roles bien definidos (2).

La realidad chilena aún muestra un escenario precario respecto a la inclusión paterna efectiva en el proceso de nacimiento (13). Aunque las políticas de protección a la primera infancia han promovido la participación paterna desde la gestación, los padres no han mostrado la adherencia esperada durante el periodo de gestación y nacimiento (14); aquellos más jóvenes y con mayor nivel de educación son los más involucrados en el proceso reproductivo y de paternidad (13). Podría pensarse que los procesos educativos de preparación para el parto dirigidos a las madres no son los indicados para mantener motivados a los padres (9), o que las metodologías utilizadas no son las adecuadas para las necesidades de aquellos.
En virtud de lo señalado, el objetivo de este estudio es explorar las necesidades educativas de los padres para implementar una preparación prenatal acorde a sus expectativas de participación en el parto y el nacimiento. Este artículo considera todo el proceso de investigación-acción (IA) que emerge de una de las categorías relacionadas con la participación de los padres en su rol de acompañante.

\section{Material y método}

Durante el periodo 2015-2016, se condujo un estudio de naturaleza cualitativa, a través del proceso de IA, vinculado a una tesis de grado del Programa de Doctorado Inter-Unidades entre dos prestigiosas universidades de la región. El propósito de utilizar la IA, sustentada en el paradigma crítico (15), fue explorar las necesidades de preparación antenatal y modificar la realidad de los participantes varones. De este modo, se desarrolló un proceso de IA en sus tres fases: exploratoria, de intervención y de evaluación, por medio de grupos focales (dos para la fase exploratoria, uno para la intervención y uno para la evaluación, cada uno de aproximadamente setenta minutos de duración).

Las tres fases de la IA conducidas por las investigadoras cumplieron con los tres objetivos de esta metodología: conocer la realidad de los participantes y levantar los temas generativos (fase exploratoria); implementar la preparación con sesiones educativas (detalladas conforme al rigor científico de la IA en la sección de resultados) (fase de intervención), y validar la intervención en conjunto investigador-participante (fase de evaluación).

Todo el proceso de investigación fue continuo, durante un año aproximadamente, y se llevó a cabo en una red de salud de la ciudad de Santiago de Chile (zona metropolitana). Esta red abarca un sistema mixto de salud, dispuesto en dos ubicaciones geográficas de la ciudad, para atender a dos segmentos de la población de distinto nivel socioeconómico. Uno de ellos corresponde al sistema público (institucionalizado); el otro, al sistema privado de atención. La captación de los participantes correspondió a la atención prenatal en ambos centros ambulatorios de esa red de atención, sin que existiera un vínculo previo entre los investigadores y los participantes. Estos accedieron a información previa del estudio por medio de afiches donde se describían el propósito y más detalles de la investigación. Los interesados se contactaron por medio de correo electrónico y teléfono con las investigadoras. 


\section{Participantes}

El grupo estuvo constituido por doce varones acompañantes de mujeres embarazadas: cinco provenientes del sistema privado, y siete provenientes del sistema público institucionalizado de atención, según un criterio de selección muestral por propósito (16). Los criterios de inclusión fueron: ser adulto; tener una pareja mayor de edad que esté cursando un embarazo fisiológico de tercer trimestre al ingreso del estudio; tener acceso a los servicios de la red de salud. El total de participantes se distribuyó en cuatro subgrupos de preparación de 2-3 varones, conforme a su incorporación según la edad gestacional.

Del primer subgrupo, surgió la necesidad de incluir en el estudio a sus parejas embarazadas como acompañantes, lo que obligó, en consonancia con el paradigma crítico y participativo, a enmendar el estudio e incluir opcionalmente la participación de aquellas. Esta alternativa se replicó de manera voluntaria en los subgrupos siguientes. Finalmente, se conformó un grupo total de doce participantes varones y once embarazadas, quienes accedieron a participar luego del proceso y firma del consentimiento informado.

\section{Protección de la identidad y confidencialidad de la información}

Todo encuentro, desde la fase exploratoria hasta las de evaluación y cierre, fue grabado en modalidad de audio MP3 y transcrito textualmente. A esto se agregaron las notas de campo y los contenidos de los cuestionarios semiestructurados. Se utilizaron nombres de fantasía para proteger la identidad de los participantes: de jugadores de futbol para los varones participantes del sistema privado, y de colores para el grupo del sistema público institucionalizado. Todos los procedimientos de investigación fueron desarrollados conforme a la Resolución 466 de 2012 do Conselho Nacional de Saúde do Brasil y de la Ley 20.120 de regulación de la investigación biomédica en Chile. Se contó, por lo tanto, con la aprobación de los comités éticos científicos universitarios de Brasil y de Chile.

\section{Resultados}

Los resultados detallados a continuación responden a la exploración de la realidad de los participantes y al análisis sistemático de la información de todas las fases de la IA. La figura 1 muestra la fuente de los resultados obtenidos del proceso, particularmente de la identificación del tema-generativo referido en este artículo (rol de acompañante), el cual, al cumplir con el criterio de rigor de confirmabilidad, fue enviado y validado por cada participante.

Las características sociodemográficas de los padres varones que participaron del proceso de IA se detallan en la tabla 1. Respecto a la edad de los varones, el promedio del total de los participantes fue de 32 años. Al observarlos por grupo, el promedio de edad en el sistema privado fue de 32 años, mientras que en el grupo del sistema institucionalizado fue de 36 años, al considerar las edades extremas de 23 y 54 años, respectivamente. Sobre el nivel de escolaridad, se observa una clara diferencia: el nivel de formación de los participantes del sistema privado de la red de salud es mayor.

Otro aspecto por destacar es la poca frecuencia de padres con experiencia previa de ser padres: solo tres participantes del total del grupo habían tenido hijos y presencia en el nacimiento.

\section{Fase exploratoria de la investigación- acción: comprensión de la realidad}

Los resultados de esta fase surgieron en torno a la reflexión y a las dinámicas desarrolladas en los grupos focales. De este modo, a partir de una dinámica, se generó el concepto de participación, al responder a la pregunta: "Para mí participar del nacimiento de mi hijo significa/ha significado...", mientras que desde la reflexión de los participantes varones, se levantaron las expectativas de participación en su rol de acompañante: "Me gustaría", "Yo espero", "Yo quiero".

El significado de la participación se relacionó con la oportunidad de estar presentes para acompañar y dar soporte efectivo al bienestar de su pareja, especialmente durante el trabajo de parto: "Participar es estar ahí en el acontecimiento, por ella y para ella". Los participantes expresaron el deseo de estar presentes y de no quedarse fuera del "acontecimiento", de ese momento maravilloso, en el que podrían acompañar a su pareja y ayudar en lo que se pueda: "Estar presente de todas maneras. Quiero estar acompañando a mi señora. Creo que es importante estar en ese momento y no estar aislado del acontecimiento porque en verdad es un acontecimiento" (Pinilla). "Estar ahí también acompañando a mi esposa porque es un momento, al fin y al cabo, maravilloso y de los dos. Quiero estar ahí y ayudarla en lo que pueda" (Messi). 
Figura 1. Fuentes de resultados del proceso investigación -acción

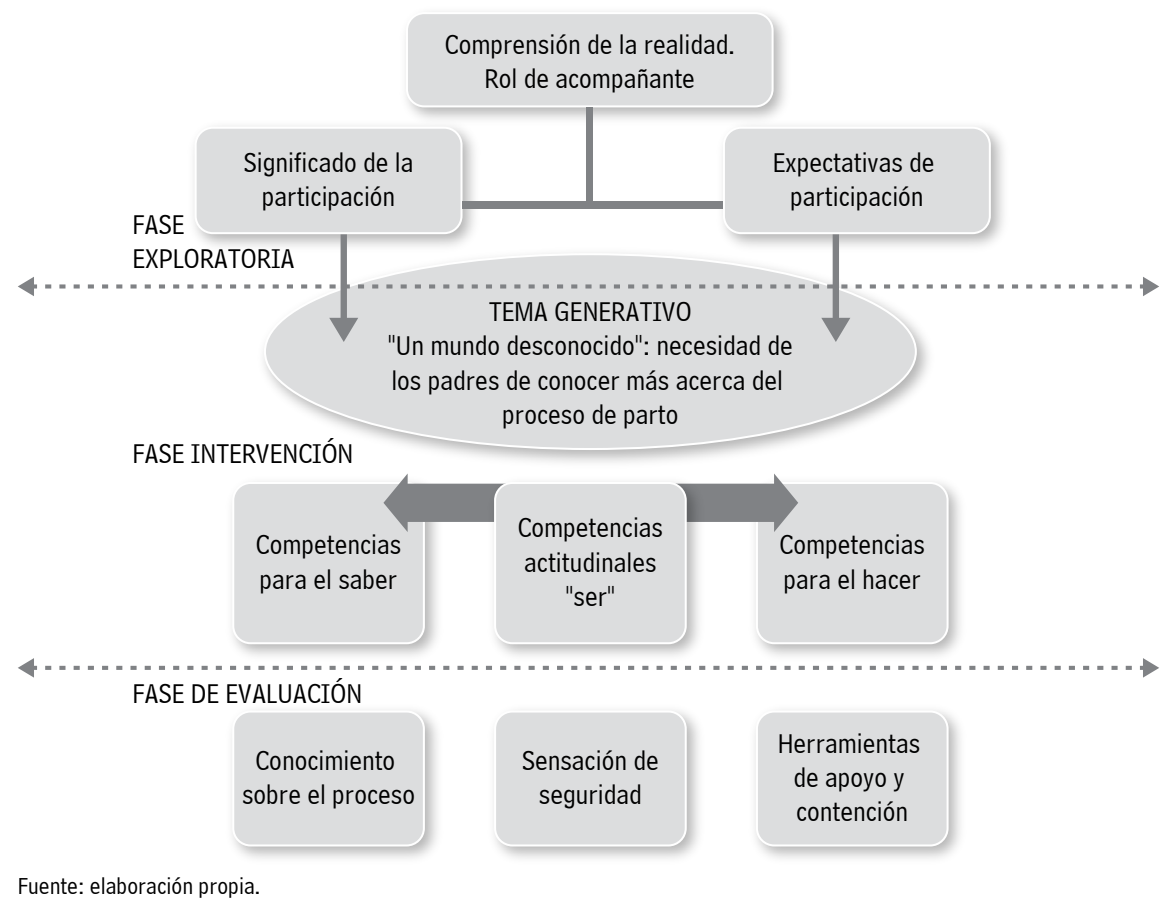

Tabla 1. Participantes por grupos y características sociodemográficas

\begin{tabular}{|c|c|c|c|c|c|}
\hline Participante & Edad & Nacionalidad & Escolaridad & Previsión & Situación de pareja \\
\hline \multicolumn{6}{|c|}{ Sistema privado } \\
\hline *Vidal & 34 & Chileno & Educación superior & Isapre & Casado \\
\hline *Medel & 35 & Chileno & Educación superior & Isapre & Casado \\
\hline *Sánchez & 29 & Chileno & Posgrado & Isapre & Casado \\
\hline$\star$ Pinilla & 32 & Chileno & Educación superior & Isapre & Casado \\
\hline *Messi & 30 & Argentino & Educación superior & Isapre & Casado \\
\hline \multicolumn{6}{|c|}{ Sistema público institucionalizado } \\
\hline *Rojo & 23 & Chileno & Educación media & Isapre & Soltero \\
\hline *Marrón & 39 & Chileno & Técnico profesional & Isapre & Casado \\
\hline *Calipso & 27 & Chileno & Educación media & Fonasa & Soltero \\
\hline *Jade & 45 & Chileno & Técnico profesional & Fonasa & Soltero \\
\hline$*_{\text {Gris }}$ & 54 & Chileno & Educación media & Fonasa & Casado \\
\hline *Sepia & 35 & Chileno & Técnico profesional & Fonasa & Soltero \\
\hline *Dorado & 31 & Chileno & Técnico profesional & Fonasa & Soltero \\
\hline
\end{tabular}

Fuente: elaboración propia. 


\section{Participar es estar ahí, por ella y para ella, de distintas formas}

En sus narrativas, los padres atribuyeron a la participación un sentido de acompañamiento y la manifestación de apoyo físico y emocional: "Dando apoyo emocional, con ciertas palabras que se pueden ocupar, masajes, hacerle cariño, cosas así. Siempre dijeron que era muy bueno para que la mujer también se relajara" (Rojo). "Estar cuando tiene las contracciones, tomarle la mano para que se sienta más acompañada, apoyándola” (Calipso). “Cómo puedo ayudar? No sé... con contención, palabras lindas, haciéndole masajes si lo necesita, sosteniéndole la mano o quedándome a un costado callado, lo que ella vaya a querer" (Messi).

Las expectativas de participación se generaron en torno a la reflexión grupal, correspondiendo principalmente a los significados atribuidos a aspectos actitudinales del "ser" de los varones como acompañantes y como soporte de su pareja.

\section{Expectativa de estar involucrado siendo útil}

El deseo de involucrarse pareciera corresponder a la apertura, motivación y sensibilidad para dar respuesta a las necesidades de la mujer y del proceso en sí mismo: "Ser un papá motivado y metido en el cuento del parto, y no solo estar ahí con cara de "Qué hago", sino saber realmente qué hacer en cada momento" (Medel). "Quiero ser útil, no quiero estar ahí como una maceta. Quiero estar ahí y ayudar en algo" (Messi). "Yo espero, en la parte previa [al parto], más que nada, ser 'el apoyo' en los momentos que haya cierta agonía o cierto agobio. Más que nada, ser apoyo para Petunia" (Gris).

\section{Expectativa de mantener el control de la situación}

En alguno de los padres se manifestó la necesidad de mantener el control para ser apoyo y aporte en situaciones límite del proceso, en las que podrían verse amenazados y superados. Esto se relaciona con uno de los aspectos más influyentes en la orientación del tema generativo:

En la etapa previa con ella, no desesperarme yo, tratar de darle calma y que la situación no me supere. Mi pareja está súper nerviosa y, como es nuestro primer hijo, ella todo lo busca. De repente se le pasa la mano y busca cosas malas en internet. Entonces siento que ella se me escapa un poco de las manos, eso no quiero, que se me escape de las manos. (Dorado)

\section{Expectativa de ser reconocido como parte del proceso}

Los padres manifestaron la necesidad de ser valorados y reconocidos en sus necesidades durante el proceso, así como de conocer los detalles prácticos que involucra su participación: "A mí que me informen cuándo puedo ir a comer. El trabajo de parto es largo, y, si uno sale, qué desubicado de mi parte estar comiendo" (Vidal) "Que no solo sea el bolsito del niño o el de la mamá, sino que también haya un bolso del papá, especial" (Medel).

\section{Tema generativo relacionado con el rol de acompañante}

A partir de la fase exploratoria, los participantes y los investigadores determinaron, en conjunto, los temas generativos. Aquel relacionado con la problemática del acompañante correspondió a "Un mundo desconocido": la necesidad de los padres de conocer más acerca del proceso del parto. Los participantes expresaron las demandas educativas con relación al desarrollo de competencias en los ámbitos del saber, del saber hacer y del ser sobre el soporte y contención en el proceso de parto. Las expresiones "cómo saber," "cómo poder" y "cómo ser" reflejaron la necesidad genuina de los padres de prepararse para acompañar integralmente a sus parejas.

\section{Competencias del saber}

Estas se relacionaron con conocer más el proceso de parto, su inicio, duración y etapas. El saber responder frente al estrés 0 ansiedad que se generan enfrente a situaciones complicadas del proceso, o simplemente saber a lo que se pueden enfrentar en una situación normal de parto: "No sé cuánto se pueda demorar un parto, pero lo que sí sé es que la dilatación de la mujer puede durar horas, puede durar mucho tiempo, entonces en realidad no todos los tiempos son iguales. Yo creo que eso sería, para empezar, saber más sobre todo eso" (Rojo). "Hay personas que quedan en shock, no saben qué hacer, no entienden nada, entonces es muy diferente decirlo. Creer cómo va a ser a vivirlo es muy diferente. Yo creo que lo que hay que desarrollar primero es a lo que uno se va a enfrentar (en el parto)" (Vidal). 


\section{Competencia del saber hacer}

Se enfocaron en la necesidad de adquirir herramientas para acompañar de forma efectiva a su pareja, física y emocionalmente, para hacer esta experiencia más sostenible para la mujer: "Cómo saber, cómo poder ayudarla para que sea más fácil vivirlo, que no sea tan pesado para ella [...] por ejemplo ciertos masajes o palabras para ayudarla" (Calipso).

\section{Competencia del ser}

En los relatos de los padres, las expresiones que revelan el sentimiento de ser padre y acompañante parecieran ser inseparables, por tratarse de una experiencia plena: "Participar activamente del nacimiento de su hijo, participar activamente en el momento del apego, pero también ser compañero de su esposa, ser apoyo para lo que ella necesite... pero también ser papá" (Sánchez).

\section{Fase de planificación e intervención de la investigación-acción: desarrollo de las sesiones educativas}

Esta fase se desarrolló con base en un trabajo colaborativo entre participantes e investigadores, según los temas generativos y sus demandas educativas. Se programaron alrededor de tres a cuatro sesiones educativas con cada subgrupo, de las cuales una estaba orientada al conocimiento sobre el proceso de parto y la presencia activa del padre. Cada sesión permitió modificar o levantar nuevas demandas, además de las establecidas en la primera fase, dado que el proceso de IA es flexible y dinámico en su totalidad (17).

La sesión educativa que correspondió a la preparación del padre en su rol de acompañante en el proceso de parto incluyó: conocer las etapas del proceso de parto; identificar las señales del inicio del trabajo de parto; comprender las diferencias entre proceso de parto y nacimiento; conocer los roles y la participación paterna en el proceso de parto y en el nacimiento; identificar y analizar las necesidades, tanto de la madre durante el proceso de parto, como del padre acompañante.

La sesión inició con un video, que cada participante presenció en silencio; esto permitió sorprender y emocionar a la audiencia: "Cuando uno lo mira, el proceso de parto tiene sus tiempos, tiene sus etapas, pero cuando uno lo mira sin estos ojos de la biología, que son muy parciales, el momento es súper mágico" (Vidal).

La segunda parte de la sesión correspondió a la dinámica de ronda de preguntas, que exploraba las percepciones de los conocimientos 0 las experiencias previas relacionadas al proceso de parto y a la participación del padre. Esta actividad en grupo se focalizó en cómo vivir dicha participación en beneficio del proceso de parto. En la reflexión por parte de los padres se habló sobre la empatía y la distancia respetuosa. Uno de los padres explicó este concepto y lo abordó desde el respeto al espacio del otro:

No estaríamos juntos si no llegáramos al punto de... por algo nos conocemos y tenemos nuestra propia dinámica, y yo respeto mucho el espacio de ella. Eso no significa que yo no esté atento a todo. También sé cuándo estar. Pese a que estoy siempre, sé cuando actuar y cuando no actuar. (Sepia)

La última parte de la sesión correspondió al aspecto práctico de la simulación del mecanismo del parto. Durante esta, los participantes tuvieron contacto con modelos fetales y pelvis maternas; tocaron al feto e intentaron movilizarlo en la pelvis hasta que saliera, simulando el mecanismo del parto.

\section{Fase de evaluación de la investigación-acción}

La fase de evaluación se consolidó en la sesión final de la intervención. Los resultados se generaron desde la reflexión grupal y desde la aplicación de un instrumento de evaluación. Los padres refirieron haber alcanzado una mayor comprensión de lo que viven la mujer y su hijo en el útero. Sin embargo, el beneficio principal fue el conocimiento que los padres y las madres adquirieron como pareja sobre las necesidades de la mujer en el parto, y sobre cómo el padre puede contribuir a su bienestar.

Un aspecto que apareció con frecuencia se relacionó con el lugar que se asignó a los padres durante todo el proceso de preparación. Los participantes sintieron que la presencia de aquel tenía un valor real para el momento del parto y del nacimiento: "[Hay que] animar al padre a tener un rol activo y a la par, tanto en el embarazo, como en el parto" (Margarita). Ellos percibían que al término del taller, habían adquirido habilidades y potenciado actitudes para enfrentar el proceso: "De una forma activa, participando en todas las etapas del parto y del nacimiento, acompañando a 
la madre, apoyándole en el parto y, posteriormente, ayudando en el cuidado del bebé" (Pinilla).

Otro aspecto relevante, y frecuente, fue el aprendizaje compartido: los participantes le asignaron un valor especial al poder compartir y aprender de las experiencias de sus pares. El hecho de no sentirse solos en situaciones 0 vivencias particulares les proporcionaba un aprendizaje con confianza y convicción. Algunos de los participantes manifestaron estas ideas como un evidente beneficio: "La experiencia de los demás participantes es un complemento de la experiencia personal" (Magnolia); "El aporte al conocimiento desde lo personal y desde las experiencias de las otras parejas" (Marrón).

Finalmente, el beneficio percibido por los padres respecto a la preparación antenatal señala un antes y un después. Aquellos manifestaron sentirse más seguros y con más herramientas para enfrentar el parto y el nacimiento: "Uno aprende eso, y debo decir que lo que hicimos en los talleres a mí me sirvió mucho [...] ni siquiera la parte conceptual, sino la parte empírica pura" (Sepia). "Uno se siente más seguro, como que me entregaron las herramientas para estar ahí, y después para poder desarrollarme más, o sea, yo le agradezco que me entregaron esta seguridad" (PiniIla). "Con seguridad y conocimiento, gracias a lo aprendido en el taller y a la reflexión que realizamos en el grupo"(Pinilla).

\section{Discusión}

Todo el proceso vivido desde la gestación hasta el nacimiento del hijo marca un hito en la vida de las madres y de los padres $(1,5,18)$. Con certeza, el acompañamiento profesional para los padres durante el periodo antenatal resulta beneficioso para el proceso, pero compartir y reflexionar en torno a sus necesidades bajo un modelo de preparación maternal puede no resultar efectivo para ellos. Así, algunos autores recomiendan desarrollar intervenciones especiales para los padres (2, 5-7).

El proceso de IA permitió desarrollar esta preparación especial para varones con un enfoque participativo $(1,19)$, lo que requiere que los padres estén involucrados en el embarazo y que deseen hacer parte del momento del nacimiento, situación poco frecuente en la actualidad, tanto en Chile, como en el resto del mundo (20). La captación y preparación de los padres varones que se distancian del proceso desde el embarazo (14), y que no toman parte en el nacimiento de su hijo (13), puede requerir inter- venciones motivacionales más complejas. Los estudios recientes demuestran que factores como el estrés, la falta de apoyo y la violación de las expectativas del padre podrían relacionarse con sentimientos negativos hacia la participación en el embarazo y el nacimiento (21), así como la sensación de ser ignorados por parte del equipo de profesionales durante todo el proceso previo al parto. Esto demuestra la necesidad de incluir preparaciones de tipo participativas y de naturaleza crítico-reflexiva (22) para usuarios y profesionales.

Bajo el perfil de motivación descrito, los varones de este estudio fueron activos participantes de las intervenciones educativas, por lo cual los resultados representan a este grupo de padres acompañantes. En el transcurso de la preparación, se demostró que los padres poseen necesidades especiales y particulares para vivir una experiencia compartida con su pareja mujer, lo que concuerda con lo reportado en otro estudio (20).

Contrario a los resultados de las encuestas nacionales y regionales, no se identificaron diferencias entre los subgrupos provenientes del sistema privado y del público respecto al conocimiento sobre el proceso de parto ni sobre las necesidades educativas que emanaron de este. Para Aguayo, sí existen diferencias respecto a la actitud de participación y de involucramiento en padres de distinto nivel de escolaridad y edad (13).

Desde la perspectiva de los contenidos de la preparación, muchos de los estudios que reportan contenidos educativos para el acompañamiento del varón se basan en actividades prácticas, como técnicas de acompañamiento para la relajación y respiración de la mujer en proceso de parto (9), pero muy poco respecto a la dimensión actitudinal. Si bien es cierto que el ser un buen acompañante para la mujer representa una necesidad relevante de preparación práctica para poder otorgar soporte físico $(8,9$, 23), este hecho de acompañar se ha generado de manera retrospectiva, con estudios cualitativos que recogen la experiencia. Este estudio, en cambio, recoge información sobre las necesidades y las expectativas de manera anticipada, lo cual podría explicar la aparición de la actitud del "ser un buen acompañante", en términos del respeto de los tiempos del proceso de parto, de la lectura que se da a las necesidades de la mujer y de la seguridad que adquieren para poder otorgar contención en momentos difíciles (24). Esto permite comprender que si las demandas educativas se exploran y se trabajan en un momento previo al nacimiento, y en 
un contexto de preparación participativa, el padre podrá manifestar sus necesidades y expectativas sobre lo que desea vivir como acompañante, más que sobre lo que le correspondió vivir, si estas se recogen una vez ocurrido el evento.

\section{Conclusión}

Este estudio permitió relevar el rol del padre y empoderarlo en su rol paterno y de acompañamiento durante el proceso de parto, pues este es un aspecto fundamental por considerar para fomentar el proceso del nacimiento en familia. Comprender la ex- periencia paterna en este evento ha fortalecido, desde la mirada disciplinar, la implementación de un modelo de cuidado integral, desarrollado en Chile desde hace una década, con resultados positivos en bienestar materno (25), pero con una comprensión limitada de la inclusión paterna. Un nacimiento que se vive en familia, y con la participación activa del padre, puede tener un impacto futuro en la salud mental y en los procesos de vinculación familiar saludable, focos relevantes del accionar de la enfermería y de su responsabilidad social.

Conflicto de interés: ninguno declarado.

\section{Referencias}

1. Manganiello A, Hoga L. Orgulho de pai : cartilha educativa para promocão do envolvimento paterno na gravidez. USP São Paulo; 2012.

2. Uribe C, Contreras A, Hoga L. Presencia activa del padre en el nacimiento integral: significados atribuidos por padres y madres a los roles paternos. Rev Chil Obs Ginecol [Internet]. 2018;83(1):22-6. DOI: 10.4067/s0717-75262018000100022

3. Hoga L, Reberte L, Higashi A, Zamo-Roth F. The experience and role of a companion during normal labor and childbirth : a systematic review of qualitative evidence. Joanna Briggs Inst Database Syst Rev. 2013;11(12):121-56. DOI: $10.11124 /$ jbisrir-2013-1178

4. Freire P. Pedagogía de la autonomia: saberes necesarios para la práctica educativa. 33. a ed. Sao Paulo: Paz y Tierra; 2004.

5. Nakano AM, Silva L, Beleza AC, Stefanello J, Gomes F. O suporte durante o processo de parturição: a visão do acompanhante. Acta Paul Enferm. 2006;20(2):131-7. DOI: 10.1590/S0103-21002007000200004

6. Premberg A, Lundgren I. Fathers' experiences of childbirth education. J Perinat Educ. 2006;15(2):21-8. DOI: $10.1624 / 105812406 \times 107780$

7. Premberg A, Carlsson G, Hellstr A, Berg M. First-time fathers' experiences of childbirth. A phenomenological study. Midwifery. 2011;27:848-53. DOI: 10.1016/j.midw.2010.09.002

8. Adeniran AS, Aboyeji AP, Fawole AA. Male partner's role during pregnancy, labour and delivery: expectations of pregnant women in Nigeria. Int J Heal Sci. 2015;9(3):305-13. Disponible en: https://www.ncbi.nlm.nih.gov/pmc/articles/ PMC4633194/

9. Erlandsson K. Prenatal parental education from the perspective of fathers with experience as primary caregiver immediately following birth: a phenomenographic study. J Perinat Educ. 2010;19(1):19-28. DOI: 10.1624/105812410X481537

10. Longworth H, Kingdon C. Fathers in the birthroom: What are they expecting and experiencing? A phenomenological study. Midwifery. 2011;27:588-94. DOI: 10.1016/j.midw.2010.06.013

11. Dolan A, Coe C. Men, masculine identities and childbirth. Sociol Heal Illn. 2011;33(7):1019-34. DOI: 10.1111/j.14679566.2011.01349.x

12. Espirito-Santo LC do, Lourenzi AB. Expectativas, sentimentos e vivências do pai durante o parto e nascimento de seu filho. Rev Gaúcha Enferm. 2000;21(2):87-109. Disponible en: https://seer.ufrgs.br/RevistaGauchadeEnfermagem/article/view/4330 
13. Aguayo F, Correa P, Cristi P. Encuesta IMAGES Chile. Resultados de la encuesta internacional de masculinidades y equidad de género. 1. ${ }^{a}$ ed. Santiago de Chile: Besegraf; 2011.

14. MIDEPLAN. Evaluación y monitoreo del Sistema Integral de Protección a la Infancia Chile Crece Contigo. Santiago Chile: s.e.; 2011.

15. Melero-Aguilar N. El paradigma crítico y los aportes de la investigación-acción participativa en la transformación de la realidad social: un análisis desde las ciencias sociales. Cuestiones Pedagógicas. 2012;21:339-55. Disponible en: http:// hdl.handle.net/11441/12861

16. Maxwell JA. Qualitative research design: an interactive approach. 3. ${ }^{\mathrm{a}}$ ed. Thousand Oaks, CA: Sage; 2013.

17. Thiollent M. Metodologia da pesquisa ação. 18. ${ }^{a}$ ed. São Paulo: Cortez; 2011:19-82.

18. Fenwick J, Bayes S, Johansson M. A qualitative investigation into the pregnancy experiences and childbirth expectations of Australian fathers-to-be. Sex Reprod Heal [Internet]. 2012;3(1):3-9. DOI: 10.1016/j.srhc.2011.11.001

19. Hoga L, Reberte L. Pesquisa-ação como estratégia para desenvolver grupo de gestantes: a percepção dos participantes. Rev Esc Enferm USP. 2007;41(4):559-66. DOI: 10.1590/S0080-62342007000400004

20. Guadagno M, Mackert M, Rochlen A. Improving prenatal health: setting the agenda for increased male involvement. AM J Mens Heal. 2013;7(6):523-6. DOI: 10.1177/1557988313490785

21. Saxbe D, Horton KT, Tsai AB. The birth experiences questionnaire: a brief measure assessing psychosocial dimensions of childbirth. J Fam Psychol. 2018;32(2):262-8. DOI: 10.1037/fam0000365

22. Franco MAS. Pedagogía da pesquisa ação. Educ e Pesqui. 2005;31(3):483-502. DOI: 10.1590/S1517-97022005000300011

23. Abushaikha L, Massah R. The roles of the father during childbirth: the lived experiences of Arab Syrian parents. Health Care Women Int [Internet]. 2011;33(2):168-81. DOI: 10.1080/07399332.2011.610534

24. Li H, Lin-Koh S, Seow L, He H-G. First-time fathers' experiences and needs during pregnancy and childbirth: a descriptive qualitative study. Midwifery [Internet]. 2014;30(6):779-87. DOI: 10.1016/j.midw.2013.10.002

25. Uribe C, Contreras A, Bravo P, Villarroel L, Abarzúa F. Modelo de asistencia integral del parto: concepto de integralidad basado en la calidad y seguridad. Rev Chil Obs Ginecol. 2018;83(3):266-76. DOI: 10.4067/s0717-75262018000300266 